\title{
Isoform Specificity in the Relationship of Actin to Dendritic Spines
}

\author{
Stefanie Kaech, Maria Fischer, Thierry Doll, and Andrew Matus \\ Friedrich Miescher Institute, 4002 Basel, Switzerland
}

\begin{abstract}
Dendritic spines contain high concentrations of actin, but neither the isoforms involved nor the mechanism of accumulation is known. In situ hybridization with specific probes established that $\beta$ - and $\gamma$-cytoplasmic actins are selectively expressed at high levels by spine-bearing neurons. Transfecting cultured hippocampal neurons with epitope-tagged actin isoforms showed that cytoplasmic $\beta$ - and $\gamma$-cytoplasmic actins are correctly targeted to spines, whereas $\alpha$-cardiac muscle actin, which is normally absent from neurons, formed aggregates in dendrites. The transfected actin cDNAs contained only coding domains, suggesting that spine targeting involves amino acid sequences in the proteins, an interpretation supported by ex-
\end{abstract}

periments with chimeric cDNAs in which C-terminal actin sequences were found to be determinative in spine targeting. By contrast to actin, microtubule components, including tubulin and MAP2, were restricted to the dendritic shaft domain. The close association of cytoplasmic actins with spines together with their general involvement in cell surface motility further supports the idea that actin motility-based changes in spine shape may contribute to synaptic plasticity.

Key words: cytoskeleton; dendrites; synapses; neuroanatomy; brain; central nervous system; microtubules; microtubuleassociated proteins; MAP2; gene expression; plasticity
The highly differentiated morphology of neurons depends in large degree on the sorting of cytoskeletal proteins to different structural microdomains within the cytoplasm (Matus et al., 1983; Ginzburg, 1991; Mandell and Banker, 1995). This phenomenon embraces each of the major cytoskeletal filament systems and in the case of actin is represented by the presence of strikingly high concentrations in dendritic spines (Matus et al., 1982; Cohen et al., 1985; Fifkova, 1985). Although the basic characteristics of cytoskeletal differentiation in neurons have been known for some years, the underlying molecular mechanisms are still poorly understood. Most of the available evidence relates to the two major neuronal microtubule-associated proteins, MAP2 and tau, which are selectively associated with dendrites and axons, respectively (for review, see Matus, 1988; Tucker, 1990). This suggests that the differing locations of some cytoskeletal proteins may depend on previous sorting of their respective messenger RNAs, because whereas MAP2 mRNA is found in dendrites, tau mRNA has been localized to the initial segment of axons (Garner et al., 1988; Litman et al., 1993). However, this is unlikely to be the only mechanism involved in determining MAP distribution, because in transgenic cells the embryonic isoform of MAP2 is sorted to dendrites although its mRNA is limited to neuronal cell bodies (Marsden et al., 1996).

In contrast to the microtubule-associated proteins, essentially nothing is known of the mechanisms that lead to the elevated concentrations of actin in dendritic spines. As with the MAPs, the potential exists for the cytoplasmic distribution of actin protein to be determined by sorting of its mRNA. In both fibroblasts and myoblasts actin mRNA sorting is isoform-specific, with $\beta$ -

Received July 28, 1997; revised Sept. 29, 1997; accepted Oct. 3, 1997.

We thank Cora Schoenenberger, Jean-Claude Perriard, Thomas Kreis, Shelley Halpain, and Lester Binder for materials, and Jan Hofsteenge for advice on protein structure assessment.

S.K. and M.F. contributed equally to this work.

Correspondence should be addressed to Dr. Andrew Matus, Friedrich Miescher Institute, P.O. Box 2543, 4002 Basel, Switzerland.

Copyright (C) 1997 Society for Neuroscience $\quad 0270-6474 / 97 / 179565-08 \$ 05.00 / 0$ cytoplasmic actin mRNA being targeted to the peripheral cytoplasm, whereas mRNAs for the $\gamma$-cytoplasmic and $\alpha$-cardiac isoforms are found only around the nucleus (Kislauskis and Singer, 1992; Hill and Gunning, 1993). This differential expression of actin isoforms has a significant impact on cell morphology (Schevzov et al., 1992; Lloyd and Gunning, 1993; Von Arx et al., 1995), in which the correct targeting of their mRNA within the cytoplasm is implicated (Kislauskis et al., 1994). In neurons the relationship of actin isoforms to the postsynaptic site is likely to have significant functional consequences, because channel activity of the NMDA-type glutamate receptor is intimately linked to actin organization (Rosenmund and Westbrook, 1993).

In view of this we have now examined actin isoform expression and distribution in neurons. In situ hybridization was used to determine which isoforms of actin are endogenously expressed in neurons and which neurons express high levels of these isoforms. To investigate the mechanism of actin targeting to dendritic spines, hippocampal neurons were transfected with cDNA constructs encoding different actin isoforms or chimeric actin sequences, each bearing a short epitope tag that allows their distribution to be determined independently of endogenous actin (Von Arx et al., 1995). Because these constructs lack the $3^{\prime}$ untranslated regions where the sequences elements that determine the distribution of actin isoform mRNAs in fibroblasts are located (Kislauskis and Singer, 1992; Hill and Gunning, 1993), they also provide a test for the potential contribution of mRNA sorting in mediating the accumulation of actin in spines. Finally, in view of the differing implications of actin filaments and microtubules for cytoskeletal dynamics and cell motility, we examined the degree of partitioning of actin and microtubule proteins between the spine and shaft domains of dendrites.

\section{MATERIALS AND METHODS}

In situ hybridization. Rats (albino, strain RA25) were deeply anesthetized by inhalation of Metofane (Mallinckrodt, Mundelein, IL) and decapitated. Brain tissues were quickly dissected and frozen directly in Cryo-embedding compound (Microm, Walldorf, Germany) cooled in 
isopentane/dry ice. Cryostat sections were cut at $12 \mu \mathrm{m}$, mounted on Superfrost/Plus glass slides (Kindler, Freiburg, Germany), and fixed with $4 \%$ paraformaldehyde in PBS, $\mathrm{pH}$ 7.4. Antisense oligodeoxynucleotide probes $33 \mathrm{bp}$ long were based on sequences in the $3^{\prime}$-UTRs of independent actin isoform cDNAs. They were labeled in parallel with ${ }^{35} \mathrm{~S}$-adenosine $5^{\prime}-[\alpha$ - thio $]$ triphosphate (NEN, DuPont, Dreiech, Germany) using terminal transferase (Sambrook et al., 1989). The sequences used were (1) rat $\gamma$-cytoplasmic actin: 5'GCGGCGATTTCTTCTTCCATTGCGATCGGCAGC-3'; (2) rat $\beta$-cytoplasmic actin: 5'-CAGCGATATCGTCATCCATGGCGAACTATCAAG-3'; and (3) rat $\alpha$-smooth muscle actin: 5'-GTCTTCCTCTTCACACATAGCTGGAGCAGCTTC-3'. Hybridization conditions and autoradiographic procedures were as described previously (Marsden et al., 1996). For nonradioactive in situ hybridization, 1125 bp from the rat $\gamma$-cytoplasmic actin cDNA sequence was cloned into the XhoI site of plasmid pcDNA3 (Invitrogen, Leek, Holland), and an antisense digoxigenin-labeled cRNA probe was prepared by in vitro transcription. The procedures used for this and the subsequent in situ hybridization were as described by Schaeren-Wiemers and GerfinMoser, (1993).

Transfection experiments. Actin cDNA sequences tagged with 11 amino acids from vesicular stomatitis virus coat protein (vsv tag) were derived from clones described in Von Arx et al. (1995). For transfection they were subcloned into a chicken $\beta$-actin promoter vector (Fregien and Davidson, 1986; Cravchik and Matus, 1993), which provides reliable neuronal expression (Marsden et al., 1996). Hippocampal cell cultures were prepared and maintained according to Goslin and Banker (1991) and transfected during preparation using DOTAP (Boehringer, Mannheim) as described by Kaech et al. (1995). To regulate the level of transgene expression, tagged constructs were diluted by the addition of vector DNA without insert. This allows expression levels to be controlled without reducing the number of cells expressing the transgene. Cells were fixed with $0.5 \%$ glutaraldehyde in microtubule stabilizing buffer (Marsden et al., 1996), treated with $0.1 \%$ sodium borohydride to quench nonspecific fluorescence, and stained for actin-vsv using monoclonal antibody against the vsv epitope (from T. Kreis, University of Geneva). Other antibodies used were monoclonal antibodies against tubulin and MAP2 (Tu27, AP14, and AP18 from L. Binder, Northwestern University), monoclonal antibody against MAP2 (HM1, Sigma, St. Louis, MO), and rabbit polyclonal antibody against MAP2 (no. 266 from S. Halpain, Scripps Institute). Rhodamine- and fluorescein-labeled second antibod-
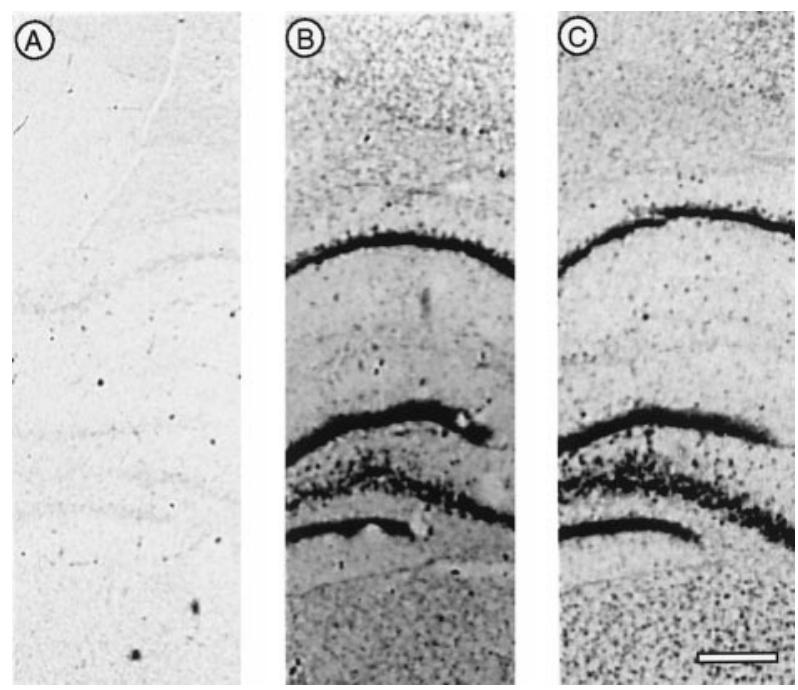

Figure 1. Differential expression of actin isoforms revealed by in situ hybridization. Radioactively labeled, isoform-specific oligodeoxynucleotide 33-mer probes were used to distinguish between $\alpha$-smooth muscle actin $(A)$ and the $\beta$ - and $\gamma$-cytoplasmic isoforms $(B, C)$. The autoradiograms are taken from neighboring frontal sections of rat forebrain that include (from top to bottom) the lower half of the cerebral cortex, the hippocampus (CA1, dentate gyrus, and CA4), and the upper portion of the thalamus. Whereas the $\alpha$-actin probe gave only background labeling, both of the cytoplasmic actins gave strong signals in cell bodies of all areas. Scale bar, $0.4 \mathrm{~mm}$. ies, cross-absorbed grade for double-label immunofluorescence, were obtained from Jackson Laboratories, (West Grove, PA). Rabbit polyclonal antibodies against the glutamate receptor GluR1 subunit were from either Anawa Biologicals (Wangen, Switzerland) or Upstate Biotechnologies (Lake Placid, NY). Appropriate dilutions for all antibodies were determined in preliminary test experiments. Staining of cells with rhodamine-phalloidin (Sigma, St. Louis, MO) was performed using a 5 $\mu \mathrm{g} / \mathrm{ml}$ solution. Coverslips containing cells transfected with GFP-tagged MAP2c (Kaech et al., 1996) were mounted in observation chambers and examined on the temperature-controlled stage of a Leica DMIRBE inverted microscope using GFP-optimized filters (Chroma Technologies, Brattleboro, VT).

Sequence alignment and structure analysis. The amino acid sequences of $\gamma$-cytoplasmic and $\alpha$-cardiac actins were aligned using a text editor. Residues where substitutions occur were converted to capital letters, and letters indicating amino acids common to both isoforms were removed by searching for lower case letters and replacing them with spaces. The two sequences were reopened in Adobe Illustrator 7.0 aligned and brought to the desired length using the kerning feature, which changes the spacing between characters evenly while preserving their relative positions. The spatial distribution of substituted residues within the three-dimensional structure of the actin molecule was assessed by inspection of the $\alpha$ skeletal muscle actin molecule. The coordinates were obtained from the Brookhaven National Laboratory Protein Data Bank (1ATN.PDB).
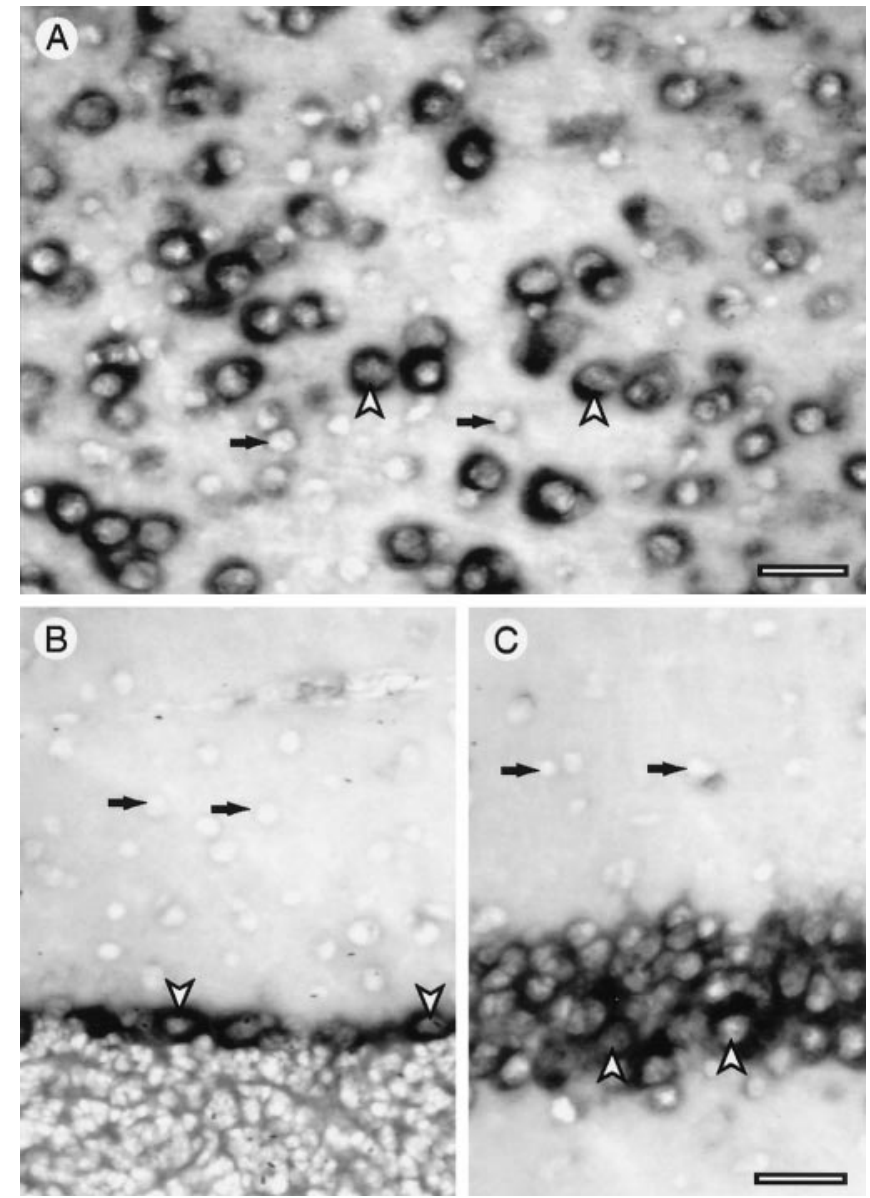

Figure 2. Cellular pattern of actin expression in brain. Sections of rat brain were processed for in situ hybridization using a digoxigenin-labeled cRNA probe for cytoplasmic actins. In the cerebral cortex $(A)$ cell bodies of pyramidal cell neurons are strongly labeled (arrowheads), whereas smaller cells, including glia and small neurons (arrows), showed weak or no signal. In the cerebellar cortex (B) Purkinje cell bodies (arrowheads) showed strong labeling, in contrast to interneurons in the molecular layer (arrows), which gave no detectable signal. In area CA1 of the hippocampus $(C)$ pyramidal neuron cell bodies are strongly labeled in contrast to cells in the neuropil, which show either no signal or a weak signal. In $A$ the pial surface is to the right. Scale bars: $A, 60 \mu \mathrm{m} ; B, C, 100 \mu \mathrm{m}$. 

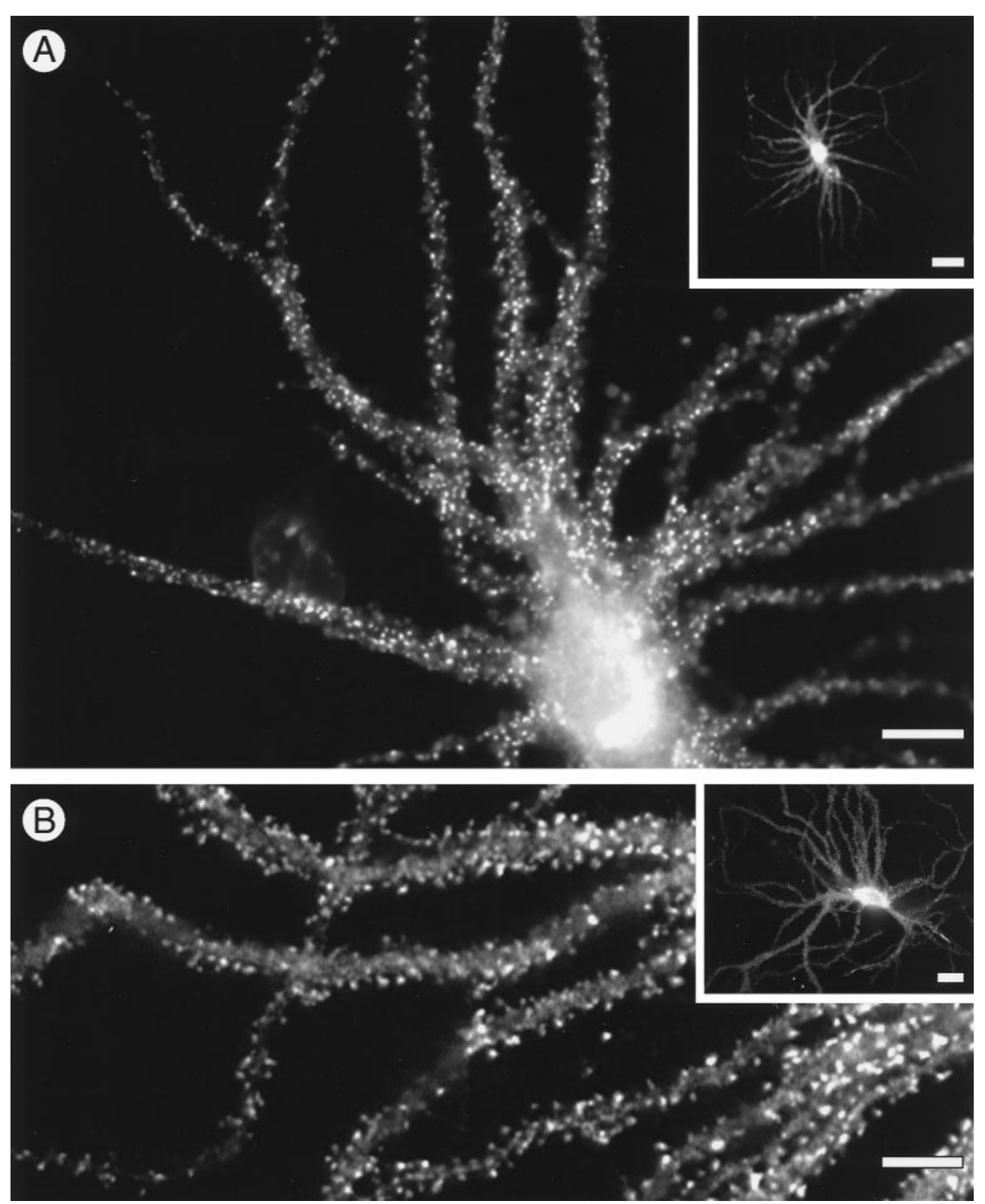

Figure 3. Targeting of transfected cytoplasmic actins to dendritic spines. Hippocampal neurons transfected with cDNA constructs expressed either $\gamma$ cytoplasmic actin $(A)$ or $\beta$-cytoplasmic actin $(B)$, which were detected by immunofluorescence staining using mouse monoclonal antibodies against the vsv epitope tags on the actin cDNAs. The accumulated actins in the dendritic spines appear as bright spots superimposed on the weakly labeled dendrites. Scale bars: $A, 20 \mu \mathrm{m}$; inset, $50 \mu \mathrm{m} ; B, 30$ $\mu \mathrm{m}$; inset, $25 \mu \mathrm{m}$.

\section{RESULTS}

\section{Endogenous expression of actin isoforms}

To determine which actin isoforms are expressed in brain neurons, rat forebrain sections were processed for in situ hybridization using ${ }^{35} \mathrm{~S}$-labeled antisense oligodeoxynucleotide probes against isoform-specific sequences in the untranslated regions of cytoplasmic or muscle actin mRNAs. Whereas muscle actin probes showed only background labeling (Fig. 1A), probes for both $\beta$ - and $\gamma$-cytoplasmic actins gave strong positive signals (Fig. $1 B, C)$. This is consistent with previous data showing that $\beta$ - and $\gamma$-cytoplasmic actins but not muscle actins are expressed in brain tissue (Chiba et al., 1990; McHugh et al., 1991). Examination of preparations made with these probes at higher magnification indicated that only large neuronal cell bodies gave strong autoradiographic signals. To confirm this and to obtain higher resolution labeling, in situ hybridization preparations were made using a digoxigenin-labeled cRNA probe for cytoplasmic actins. This revealed that in various brain areas high level expression of cytoplasmic actin mRNA was limited to large neurons (Fig. 2). In the cerebral cortex, pyramidal neurons in layers IV and $\mathrm{V}$ were strongly labeled (Fig. $2 A$, arrowheads), whereas glial cell bodies and small neurons (Fig. $2 A$, black arrows) showed weak or no labeling. A similar situation was found in the cerebellar cortex, where only Purkinje cells were conspicuously labeled (Fig. 2B), and in the hippocampus, where pyramidal cells in the CA1 area were strongly labeled (Fig. 2C). Note, however, the low but distinct signals present in some interneurons (e.g., Fig. $2 C$, righthand arrow).

\section{Transfection experiments}

The relationship of individual actin isoforms to neuronal structure was investigated by transfecting cultured hippocampal neurons with constructs expressing vsv-tagged actin isoforms. After 4 to 5 weeks in culture, such cells express large numbers of spineshaped protuberances whose identity as spine synapses has previously been confirmed by electron microscopy (Bartlett and 

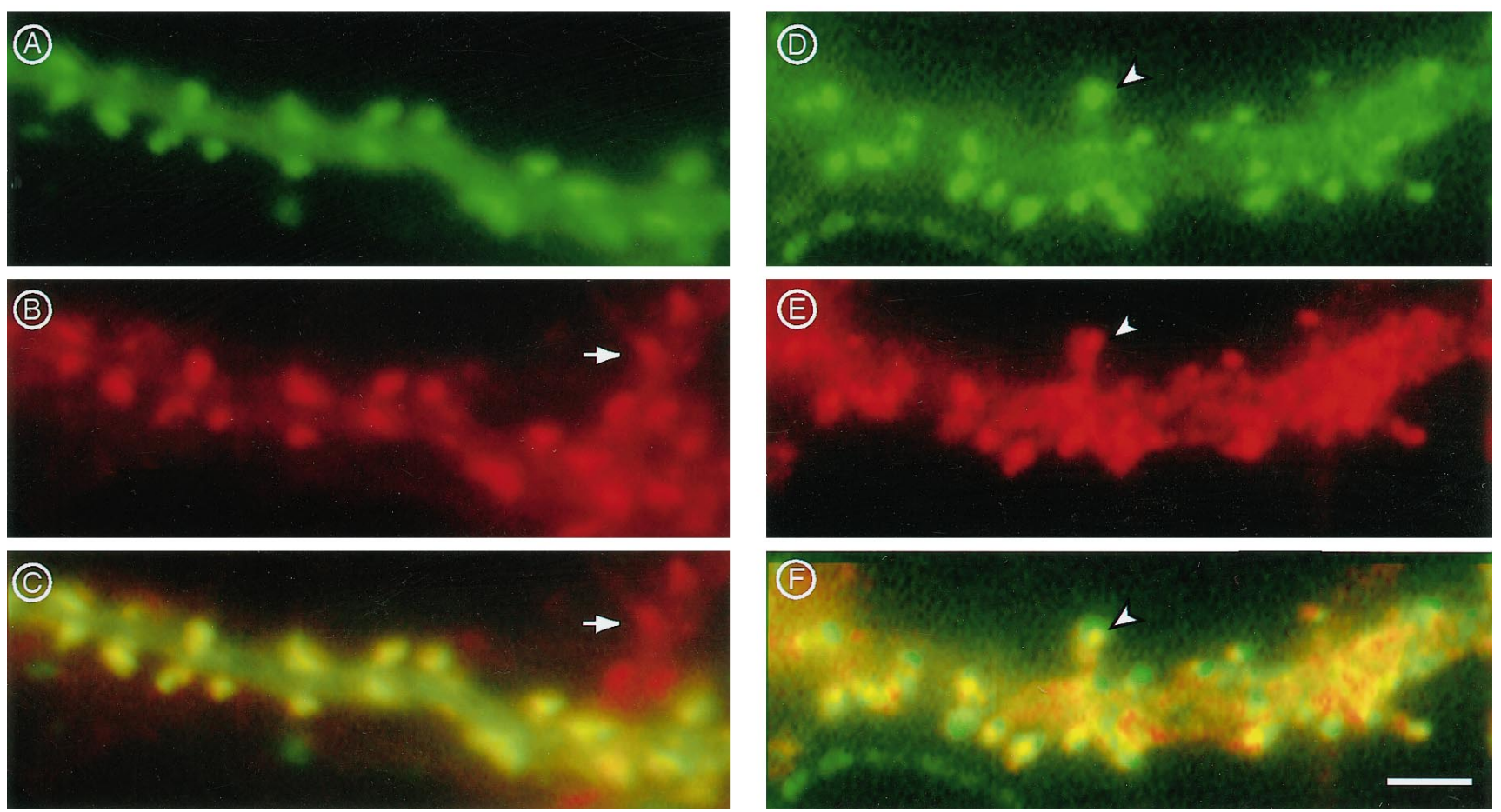

Figure 4. Colocalization of actin expressed by transfection with endogenous actin and glutamate receptor proteins. $A$, Part of a dendrite of a transfected neuron showing the distribution of transgenically expressed $\gamma$-cytoplasmic actin revealed by immunostaining with monoclonal antibody against the vsv epitope tag compared with the distribution of endogenous filamentous actin revealed by staining with rhodamine-labeled phalloidin $(B)$. The combined labeling pattern is shown in $C$. The arrow indicates part of a dendrite from a neighboring untransfected neuron. $D$, Part of the dendrite of a transfected cell in another culture that was stained for actin-vsv and with antibody against the GluR1 subunit of the AMPA receptor $(E)$. Superimposition of the two patterns $(F)$ reveals heterogeneity of GluR1 levels in various spines (one example is indicated by the arrowhead) and shows receptors located on the dendritic shaft (scattered red immunofluorescence). Scale bar, $1.5 \mu \mathrm{m}$.

Banker, 1984). Cultures transfected with vsv-labeled actins were fixed and stained with monoclonal antibodies against the vsv tag, which revealed individual cells expressing the transgene protein (Fig. 3). Both $\beta$ - and $\gamma$-cytoplasmic actins were highly concentrated in dendritic spines (Fig. $3 A, B$ ). For both isoforms, anti-vsv staining was also present in the cytoplasm of dendritic and axonal processes but at levels much below those in dendritic spines.

The association of transfected cytoplasmic actins with dendritic spines was confirmed by examining cultures that were doublelabeled with monoclonal antibodies against the vsv tag of the transfected actin and either rhodamine-phalloidin or polyclonal rabbit antibodies against the GluR1 subunit of the AMPA-type glutamate receptor (Fig. 4). Staining for transfected actin using antibodies against the vsv tag (Fig. $4 A$ ) showed a pattern closely similar to rhodamine-phalloidin staining for total actin (Fig. 4B). This was particularly evident when the two staining patterns were superimposed: yellow coloration indicates overlap between the two staining patterns (Fig. $4 C$ ). Whereas rhodamine-phalloidin staining was essentially limited to spines, actin-vsv staining was also present in dendritic shafts, although at lower levels than in spines. This is probably because rhodamine-phalloidin labels only

$\rightarrow$

Figure 5. Distribution of $\alpha$-cardiac actin in transfected cells. $A$, Staining with anti-vsv antibodies shows the formation of elongated deposits in dendritic shaft cytoplasm of transfected cells. $B$, A section of dendrite double-stained for actin with anti-vsv (fluorescein, green) and for the GluR1 glutamate receptor subunit (rhodamine, red). The presence of exogenous $\alpha$-cardiac actin in the dendritic shaft does not interfere with the formation of spines, which are revealed by their content of GluR1 protein. Scale bars: $A, 70 \mu \mathrm{m} ; B, 1.5 \mu \mathrm{m}$. filamentous actin, whereas the anti-vsv staining reveals vsv-tagged actin in both actin filaments and in the pool of unpolymerized monomeric actin, suggesting that the low levels of actin in dendritic shafts are primarily monomeric. Rhodamine-phalloidin la-
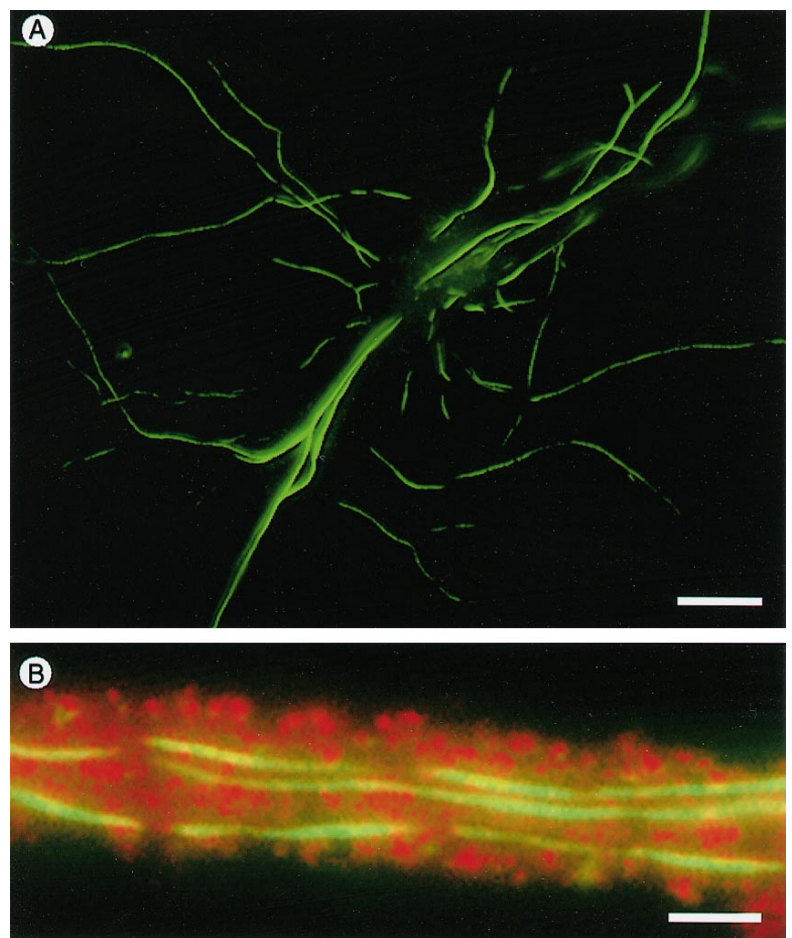
beling was not significantly stronger in spines of actin-vsvexpressing cells than in spines of untransfected cells (e.g., as indicated by the arrows in Fig. 4B,C), indicating that actin-vsv in transfected cells was expressed at trace levels.

Overlapping patterns were also found for double-labeling of actin-vsv and the GluR1 subunit of the AMPA-type glutamate receptor (Fig. $4 D-F$ ). In this case fluorescent signal levels from actin-vsv and the GluR1 receptor in individual spines were not necessarily the same (Fig. 4D-F, arrowheads). Furthermore GluR1 labeling was more widespread than actin-vsv, especially along the dendritic shaft (Fig. $4 F$ ). This probably reflects the presence of glutamate receptors at synapses that are not associated with spines.

In contrast to the cytoplasmic actins, $\alpha$-cardiac muscle actin characteristically formed cylindrical deposits in dendritic shafts and were only rarely present in spines (Fig. 5). Expression of $\alpha$-cardiac actin did not noticeably impair cell viability or process formation, even after 5 weeks in culture (Fig. $5 A$ ), nor did it appear to interfere with the formation of spines, which could be visualized in $\alpha$-actin-expressing cells by double-labeling with anti-vsv for actin and anti-GluR1 for glutamate receptors, including those located on spines (Fig. 5B).

To investigate the sequence dependence of actin isoform targeting to spines, cells were transfected with chimeric actin constructs containing either the $\mathrm{N}$-terminal 83 amino acids of $\alpha$-cardiac actin linked to the $\mathrm{C}$ terminus of $\gamma$-cytoplasmic $(\alpha, \gamma$ actin) or the opposite conformation ( $\gamma, \alpha$-actin) (see Fig. 8A). $\alpha, \gamma$-actin, containing the $\mathrm{C}$-terminal domain of $\gamma$-cytoplasmic actin, was present at high concentrations in spines in a pattern indistinguishable from that of $\gamma$-cytoplasmic actin itself (Fig. 6A). By contrast, the opposite construct in which the N-terminal 83 amino acids of $\gamma$-cytoplasmic actin were linked to the $\mathrm{C}$ terminus of $\alpha$-cardiac actin showed the same pattern as $\alpha$-cardiac actin, with long cylindrical deposits in the cytoplasm of the dendritic shaft and few labeled spines (Fig. 6B).

\section{Comparative distributions of cytoplasmic actins and microtubule proteins}

The relative distributions of actin and microtubule proteins in relation to dendritic structure were also examined using transfected cells. Figure 7 shows the pattern of protein distribution in $\gamma$-cytoplasmic actin-vsv transfected cells that were doubleimmunofluorescence-labeled for actin, using anti-vsv, and for endogenous MAP2. Figure $7 B$ shows part of the dendrites from two neighboring cells in the same culture, one of which is from a transfected cell expressing actin-vsv and the other from a neighboring untransfected cell. Both dendrites show rhodamine staining along their length for endogenous MAP2, which forms a distinct contrast to the intense anti-vsv fluorescein staining for $\gamma$-actin in dendritic spines. This contrasting distribution of actin and MAP2 was a consistent feature of spine-bearing cells in cultures transfected with either $\beta$ - or $\gamma$-actin-vsv; actin was highly concentrated in spines, whereas MAP2 was abundant along the entire length of shafts and was never detectable in spines. To be sure that this did not result from masking of a single MAP2 epitope in dendritic spines, we stained cultures with several MAP2 antibodies directed against different epitopes (see Materials and Methods). None of these stained dendritic spines. As a further test of MAP2 distribution in dendrites we transfected hippocampal neurons with MAP2 tagged with green fluorescent protein (MAP2-GFP), which allows the distribution of MAP2 in living cells to be visualized (Kaech et al., 1996). Like endogenous
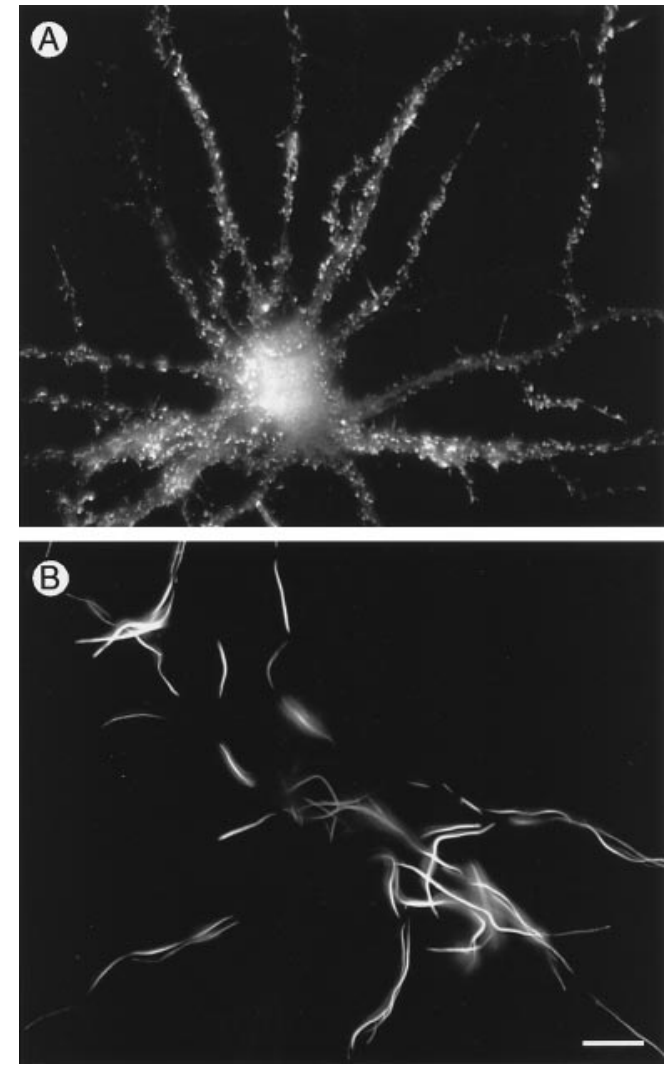

Figure 6. Cytoplasmic distribution of chimeric actins in transfected neurons. Cells expressing vsv-tagged chimeric actin cDNAs consisting of either the $\mathrm{N}$-terminal 83 amino acids of $\alpha$-cardiac muscle actin joined to the $\mathrm{C}$ terminus of $\gamma$-cytoplasmic actin $(A)$ or the $\mathrm{N}$-terminal 83 amino acids of $\gamma$-cytoplasmic actin joined to the $\mathrm{C}$ terminus of $\alpha$-cardiac muscle actin $(B)$. Whereas $\alpha, \gamma$-actin targets normally to dendritic spines $(A)$, $\gamma, \alpha$-actin does not enter spines but forms the long cylindrical deposits in dendritic shafts characteristic of $\alpha$-cardiac actin $(B)$. Scale bar, $40 \mu \mathrm{m}$.

wild-type MAP2, MAP2-GFP expressed in neurons is found only in dendrites where it is limited to the shaft domain and is excluded from dendritic spines (Fig. 7C).

\section{DISCUSSION}

The experiments reported here show that cytoplasmic actin isoforms are endogenously expressed at high levels in spine-bearing neurons in various brain areas. Transfection experiments using cultured hippocampal neurons showed that whereas these cytoplasmic actins accumulate in spines, $\alpha$-cardiac muscle actin, which is not normally expressed in brain, does not enter spines.

Our results also demonstrate a striking partitioning of the dendritic cytoplasm into an actin-rich spine domain and a shaft domain dominated by microtubules. Whereas phalloidin-stained endogenous actin and transfected cytoplasmic actins were concentrated in spines, tubulin and MAP2 were limited to the dendrite shaft. The exclusion of MAP2 from dendritic spines was shown by staining cultured hippocampal neurons with several MAP2 antibodies, both monoclonal and polyclonal, directed against different epitopes, and by the distribution of GFP-tagged MAP2 in living cells. This differential distribution of actin and microtubule proteins is consistent with observations from electron microscopy showing that whereas microtubules form dense fascicles in the shafts of dendrites, they are absent from dendritic spines (Gray, 1959; Peters et al., 1976; Westrum et al., 1980). A 

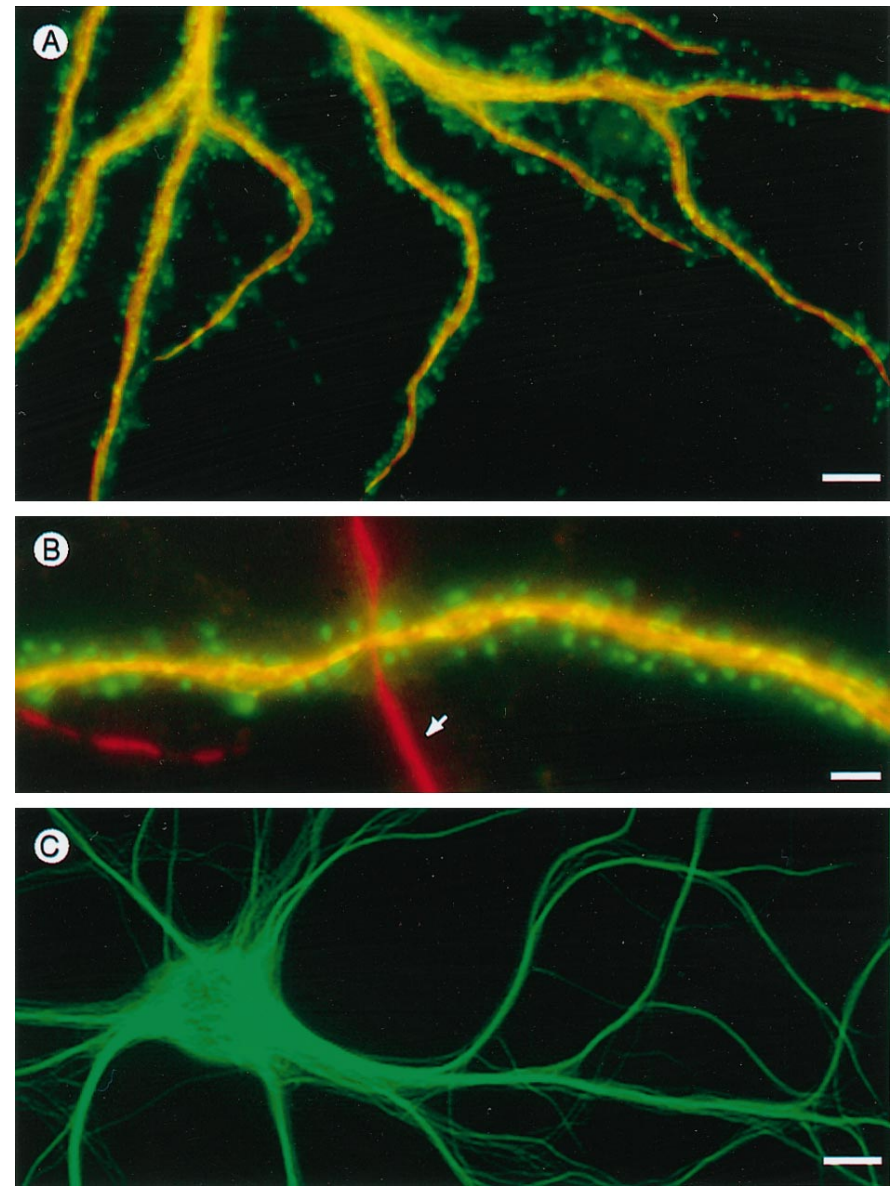

Figure 7. Partitioning of actin and microtubule-associated protein MAP2 in dendrites. $A$, Hippocampal cell transfected with vsv-tagged $\gamma$-cytoplasmic actin, fixed with glutaraldehyde, and stained with anti-vsv for actin (fluorescein channel) and MAP2 (rhodamine channel). B, A second example showing portions of a dendrite from a transfected cell (horizontal) crossed by a dendrite from a neighboring nontransfected cell (vertical, arrow). Note the contrasting pattern of $\gamma$-cytoplasmic actin in spines compared with MAP2 in the dendritic shaft domain. $C$, Dendrites of a living hippocampal neuron expressing GFP-tagged MAP2, which is abundant in the shaft domain but absent from dendritic spines. Scale bars: $A, 10 \mu \mathrm{m} ; B, 1.5 \mu \mathrm{m} ; C, 8 \mu \mathrm{m}$.

notable exception to this occurs in the unusually large branched spines on CA3 pyramidal cells where microtubules are clearly visible (Chicurel and Harris, 1992). The presence of clearly distinguishable microtubules in these large spines underlines their absence from the vast majority of smaller spines found elsewhere. Rather than microtubules, the spine cytoplasm is characterized by the presence of fine filaments (Gray, 1959; Peters et al., 1976; Landis and Reese, 1983), which immunocytochemistry and meromyosin labeling have shown to contain actin (Katsumaru et al., 1982; Cohen et al., 1985; Markham and Fifkova, 1986).

Past studies have reported the presence of MAP2 immunoreactivity in spines (Caceres et al., 1984; Morales and Fifkova, 1989), but more recent data suggest that this is probably an artifact of formaldehyde fixation. MAP2 binds tightly to microtubules in living transfected non-neuronal cells (Kaech et al., 1996), but whereas glutaraldehyde fixation correctly localizes MAP2 attached to microtubules (Weisshaar et al., 1992), formaldehyde fixation produces diff use staining that is spread through- out the cytoplasm (B. Weisshaar and A. Matus, unpublished observations). Correspondingly, when cultured cells are fixed with glutaraldehyde, as here, spines are never stained by MAP2 antibodies, whereas after formaldehyde fixation we also observe MAP2 immunoreactivity in spines (our unpublished observations). Actin is generally associated with cell surface motility, whereas microtubules are considered to impart morphological stability, a distinction that is particularly prominent at the transition zone between growth cone and process in developing neurites (Forscher and Smith, 1988; Tanaka and Sabry, 1995). The extreme partitioning of actin in spines and microtubule proteins in the shafts of dendrites thus suggests a substantial functional subdivision in the dendritic cytoplasm.

The results of in situ hybridization labeling using isoformspecific oligodeoxynucleotide probes are consistent with previous reports (Chiba et al., 1990; McHugh et al., 1991) that brain tissues express only cytoplasmic actin isoforms. In all brain areas examined, strong in situ hybridization signals for actin mRNA were selectively associated with large neurons, such as cortical pyramidal cells and cerebellar Purkinje cells, linking high levels of actin expression to spine production. Hybridization signals for actin mRNAs were strong in cell bodies but undetectable in dendrites, suggesting that actin targeting to dendritic spines is determined by sequences in the protein rather than the mRNA. This is also in accordance with the absence from our transfected constructs of the $3^{\prime}$-untranslated cytoplasmic actin sequences that guide the sorting of cytoplasmic actin isoform mRNAs in non-neuronal cells (Hill and Gunning, 1993; Kislauskis et al., 1993). The involvement of protein rather than mRNA in determining actin targeting to spines is also suggested by the finding that both $\beta$ and $\gamma$-cytoplasmic actins accumulate in spines, whereas in nonneuronal cells the mRNAs for these isoforms have different distributions (Hill and Gunning, 1993). The dependence of spine accumulation on protein rather than mRNA sequence is also indicated by experiments with chimeric actin cDNAs, which showed a requirement for the $\mathrm{C}$ terminus of cytoplasmic actin. In the 292 amino acids of this $\mathrm{C}$-terminal domain the $\beta$ - and $\gamma$ cytoplasmic sequences are identical and differ from those of $\alpha$-cardiac actin by only 13 scattered residues (Fig. $8 B$ ). Inspection of the relative positions of these residues using three-dimensional structural data showed that they are widely distributed within the actin molecule (J. Hofsteenge and A. Maus, unpublished observations). Many cases in which protein organization depends on amino acid sequence are dependent on distinct motifs within a limited area. The scattered nature of the dendritic targeting signal in the cytoplasmic actins may indicate an effect of one or more residues in regulating the folding of the $\mathrm{C}$ terminus in a way that influences its interaction with other proteins. Alternatively, it may indicate that to be functionally effective a motif need not be compact but may have its sequence characteristics scattered throughout the functional domain. This could have significant implications for both the definition and the recognition of protein interaction sequence motifs.

There are several means by which cytoplasmic actins might accumulate in spines, the most obvious being via a direct association with other components within the spine itself. A plausible location for these is the postsynaptic density (PSD), the junctional organelle characteristic of the dendritic component of axospinous synapses (Gray, 1959). PSDs contain high levels of actin (Banker et al., 1974; Cohen et al., 1977; Matus et al., 1982), which are present as filaments anchored to the PSD and running through the cytoplasm of the spine (Cohen et al., 1985; Markham 
(A)

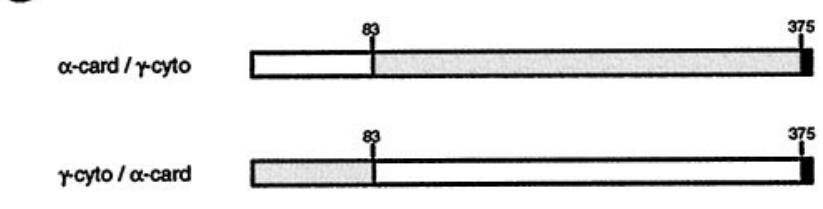

(B)
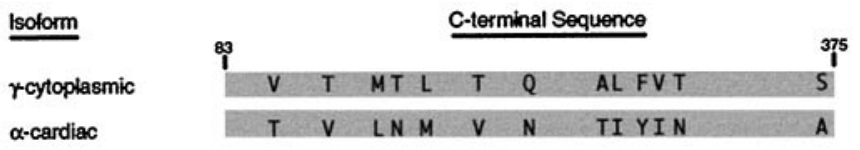

Figure 8. Diagrammatic summary of amino acid differences between cytoplasmic and muscle actin isoforms related to the differential targeting of actin to dendritic spines. Small numbers above the diagrams indicate residue numbers. $A$, Diagrams of the two chimeric isoforms used in transfection studies. $\gamma$-cytoplasmic actin sequences are shown as gray boxes, $\alpha$-cardiac actin sequences by white boxes, and the vsv epitope tag by the short black box. The upper construct targets correctly to spines, whereas the lower construct does not (Fig. 6). B, Diagrams of the Cterminal portions of the $\gamma$-cytoplasmic and $\alpha$-cardiac actin actin sequences with amino acid substitutions indicated at their correct relative positions.

and Fifkova, 1986). This actin is relatively loosely attached to the PSD structure and can be extracted under conditions that do not remove either glutamate receptor protein or another major PSD protein, the $\alpha$-subunit of calcium-calmodulin kinase II (Adam and Matus, 1996). Recently it was reported that one form of the actin-bundling protein $\alpha$-actinin binds to protein subunits of the NMDA glutamate receptor through specific sequences and that this interaction is reversible under the influence of calcium and calmodulin (Wyszynski et al., 1997). The formation of such intermolecular complexes suggests one possible explanation for the association of actin filaments with the PSD and hence the concentration of actin filaments in the spine cytoplasm. It also suggests a potential mechanism for reversibly coupling actin filaments to the postsynaptic site. The state of actin polymerization at postsynaptic sites has been shown to be closely and specifically coupled to the channel activity of NMDA-type glutamate receptors (Rosenmund and Westbrook, 1993), raising the possibility that the isoform-dependent actin targeting described here plays a significant role in the functional organization of excitatory synapses in the brain.

\section{REFERENCES}

Adam G, Matus A (1996) Role of actin in the organisation of brain postsynaptic densities. Mol Brain Res 43:246-250.

Banker G, Churchill L, Cotman CW (1974) Proteins of the postsynaptic density. J Cell Biol 63:456-465.

Bartlett WP, Banker GA (1984) An electron microscopic study of the development of axons and dendrites by hippocampal neurons in culture II. Synaptic relationships. J Neurosci 4:1954-1965.

Caceres A, Binder LI, Payne MR, Bender P, Rebhun L, Steward O (1984) Differential localization of tubulin and microtubule-associated protein 2 MAP2 in brain tissue as revealed by immunocytochemistry with monoclonal hybridoma antibodies. J Neurosci 4:394-410.

Chiba T, Nakamura Y, Sakiyama S (1990) Developmental changes of expression of non-muscle (beta and gamma) actin mRNAs in the central nervous system studied by in situ hybridization. Neurosci Lett 112:31-36.

Chicurel ME, Harris KM (1992) Three-dimensional analysis of the structure and composition of CA3 branched dendritic spines and their synaptic relationships with mossy fiber boutons in the rat hippocampus. J Comp Neurol 325:169-182.

Cohen RS, Blomberg F, Berzins K, Siekevitz P (1977) The structure of postsynaptic densities isolated from dog cerebral cortex. I. Overall morphology and protein composition. J Cell Biol 74:181-203.

Cohen RS, Chung SK, Pfaff DW (1985) Immunochemical localization of actin in dendritic spines of the cerebral cortex using colloidal gold as a probe. Cell Mol Neurobiol 5:271-284.

Cravchik A, Matus A (1993) A novel strategy for the immunological tagging of cDNA constructs. Gene 137:139-143.

Fifkova E (1985) Actin in the nervous system. Brain Res 9:187-215.

Forscher P, Smith SJ (1988) Actions of cytochalasins on the organization of actin filaments and microtubules in a neuronal growth cone. J Cell Biol 107:1505-1516.

Fregien N, Davidson N (1986) Activating elements in the promoter region of the chicken beta-actin gene. Gene 48:1-11.

Garner CC, Tucker RP, Matus A (1988) Selective localization of messenger RNA for cytoskeletal protein MAP2 in dendrites. Nature 336:674-677.

Ginzburg I (1991) Neuronal polarity: targeting of microtubule components into axons and dendrites. Trends Biochem Sci 16:257-261.

Goslin K, Banker G (1991) Rat hippocampal neurons in low-density culture. In: Culturing nerve cells (Goslin K, Banker G, eds), pp 252282. Cambridge, MA: MIT.

Gray EG (1959) Axo-somatic and axo-dendritic synapses of the cerebral cortex. An electron microscope study. J Anat 93:420-433.

Hill MA, Gunning P (1993) Beta and gamma actin mRNAs are differentially located within myoblasts. J Cell Biol 122:825-832.

Kaech S, Kim JB, Cariola M, Ralston E (1995) Improved lipid-mediated gene transfer into primary cultures of hippocampal neurons. Mol Brain Res 35:344-348.

Kaech S, Ludin B, Matus A (1996) Cytoskeletal plasticity in cells expressing neuronal microtubule-associated proteins. Neuron 17:1189-1199.

Katsumaru H, Murakami F, Tsukahara N (1982) Actin filaments in dendritic spines of red nucleus neurons demonstrated by immunoferritin localization and heavy meromyosin binding. Biomed Res 3:337-340.

Kislauskis EH, Singer RH (1992) Determinants of mRNA localization. Curr Opin Cell Biol 4:975-978.

Kislauskis EH, Li Z, Singer RH, Taneja KL (1993) Isoform-specific $3^{\prime}$-untranslated sequences sort $\alpha$-cardiac and $\beta$-cytoplasmic and actin messenger RNAs to different cytoplasmic compartments. J Cell Biol 123:165-172.

Kislauskis EH, Zhu X, Singer RH (1994) Sequences responsible for intracellular localization of beta-actin messenger RNA also affect cell phenotype. J Cell Biol 127:441-451.

Landis DM, Reese TS (1983) Cytoplasmic organization in cerebellar dendritic spines. J Cell Biol 97:1169-1178.

Litman P, Barg J, Rindzoonski L, Ginzburg I (1993) Subcellular localization of tau mRNA in differentiating neuronal cell culture: implications for neuronal polarity. Neuron 10:627-638.

Lloyd C, Gunning P (1993) Noncoding regions of the gamma-actin gene influence the impact of the gene on myoblast morphology. J Cell Biol 121:73-82.

Mandell JW, Banker GA (1995) The microtubule cytoskeleton and the development of neuronal polarity. Neurobiol Aging 16:229-237.

Markham JA, Fifkova E (1986) Actin filament organization within dendrites and dendritic spines during development. Brain Res 392:263-269.

Marsden KM, Doll T, Ferralli J, Botteri F, Matus A (1996) Transgenic expression of embryonic MAP2 in adult mouse brain: implications for neuronal polarization. J Neurosci 16:3265-3273.

Matus A (1988) Microtubule-associated proteins: their potential role in determining neuronal morphology. Annu Rev Neurosci 11:29-44.

Matus A, Ackermann M, Pehling G, Byers HR, Fujiwara K (1982) High actin concentrations in brain dendritic spines and postsynaptic densities. Proc Natl Acad Sci USA 79:7590-7594.

Matus A, Huber G, Bernhardt R (1983) Neuronal microdifferentiation. Cold Spring Harb Symp Quant Biol 48:775-782.

McHugh KM, Crawford K, Lessard JL (1991) A comprehensive analysis of the developmental and tissue-specific expression of the isoactin multigene family in the rat. Dev Biol 148:442-458.

Morales M, Fifkova E (1989) In situ localization of myosin and actin in dendritic spines with the immunogold technique. J Comp Neurol 279:666-674.

Peters A, Palay SL, Webster HF (1976) The fine structure of the nervous system. New York: Oxford UP. 
Rosenmund C, Westbrook GL (1993) Calcium-induced actin depolymerization reduces NMDA channel activity. Neuron 10:805-814.

Sambrook J, Fritsch EF, Maniatis T (1989) Molecular cloning: a laboratory manual, Ed 2. Cold Spring Harbor, NY: Cold Spring Harbor Laboratory.

Schaeren-Wiemers N, Gerfin-Moser A (1993) A single protocol to detect transcripts of various types and expression levels in neural tissue and cultured cells: in situ hybridization using digoxigenin-labelled cRNA probes. Histochemistry 100:431-440.

Schevzov G, Lloyd C, Gunning P (1992) High level expression of transfected b- and $\gamma$-actin genes differentially impacts on myoblast cytoarchitecture. J Cell Biol 117:775-785.

Tanaka E, Sabry J (1995) Making the connection: cytoskeletal rearrangements during growth cone guidance. Cell 83:171-176.
Tucker RP (1990) The roles of microtubule-associated proteins in brain morphogenesis: a review. Brain Res Rev 15:101-120.

Von Arx P, Bantle S, Soldati T, Perriard J-C (1995) Dominant negative effect of cytoplasmic actin isoproteins on cardiomyocyte cytoarchitecture and function. J Cell Biol 131:1759-1773.

Weisshaar B, Doll T, Matus A (1992) Reorganisation of the microtubular cytoskeleton by embryonic microtubule-associated protein 2 (MAP2c). Development 116:1151-1161.

Westrum LE, Jones DH, Gray EG, Barron J (1980) Microtubules, dendritic spines and spine apparatuses. Cell Tissue Res 208:171-181.

Wyszynski M, Lin J, Rao A, Nigh E, Beggs AH, Craig AM, Sheng M (1997) Competitive binding of alpha-actinin and calmodulin to the NMDA receptor. Nature 385:439-442. 